\title{
Association of Polypharmacy and Potentially Inappropriate Medications With Physical Functional Impairments in Older Adults With Cancer
}

Mostafa R. Mohamed, MBBCh, MSc $c^{1, *}$; Erika Ramsdale, MD ${ }^{1, *}$; Kah Poh Loh, MBBCh ${ }^{1}$; Huiwen Xu, PhD ${ }^{1}$; Amita Patil, MS ${ }^{1}$; Nikesha Gilmore, $\mathrm{PhD}^{1}$; Spencer Obrecht, MS ${ }^{1}$; Megan Wells, $\mathrm{MPH}^{1}$; Ginah Nightingale, PharmD ${ }^{2}$; Katherine M. Juba, $\mathrm{PhD}^{3}$; Bryan Faller, MD; Adedayo Onitilo, MD; Thomas Bradley, MD; Eva Culakova, PhD; Holly Holmes, MD; and Supriya G. Mohile, MD ${ }^{1}$

\section{ABSTRACT}

Background: Polypharmacy and potentially inappropriate medications (PIMs) are prevalent in older adults with cancer, but their associations with physical function are not often studied. This study examined the associations of polypharmacy and PIMs with physical function in older adults with cancer, and determined the optimal cutoff value for the number of medications most strongly associated with physical functional impairment. Methods: This cross-sectional analysis used baseline data from a randomized study enrolling patients aged $\geq 70$ years with advanced cancer starting a new systemic cancer treatment. We categorized PIM using 2015 American Geriatrics Society Beers Criteria. Three validated physical function measures were used to assess patient-reported impairments: activities of daily living (ADL) scale, instrumental activities of daily living (IADL) scale, and the Older Americans Resources and Services Physical Health (OARS PH) survey. Optimal cutoff value for number of medications was determined by the Youden index. Separate multivariate logistic regressions were then performed to examine associations of polypharmacy and PIMs with physical function measures. Results: Among 439 patients (mean age, 76.9 years), the Youden index identified $\geq 8$ medications as the optimal cutoff value for polypharmacy; $43 \%$ were taking $\geq 8$ medications and $62 \%$ were taking $\geq 1$ PIMs. On multivariate analysis, taking $\geq 8$ medications was associated with impairment in $\mathrm{ADL}$ (adjusted odds ratio [aOR], 1.64;95\% Cl, 1.01-2.58) and OARS PH (aOR, 1.73; 95\% Cl, 1.01-2.98). PIMs were associated with impairments in IADL (aOR, 1.72; 95\% Cl, 1.09-2.73) and OARS PH (aOR, 1.97; 95\% Cl, 1.15-3.37). A cutoff of 5 medications was not associated with any of the physical function measures. Conclusions: Physical function, an important component of outcomes for older adults with cancer, is cross-sectionally associated with polypharmacy (defined as $\geq 8$ medications) and with PIMs. Future studies should evaluate the association of polypharmacy with functional outcomes in this population in a longitudinal fashion.

J Natl Compr Canc Netw 2021;19(3):267-274 doi: $10.6004 /$ jnccn.2020.7628

\footnotetext{
${ }^{1}$ James P. Wilmot Cancer Center, University of Rochester Medical Center, Rochester, New York; ${ }^{2}$ Department of Pharmacy Practice, Thomas Jefferson University, Philadelphia, Pennsylvania; ${ }^{3}$ Department of Pharmacy, University of Rochester Medical Center, Rochester, New York; ${ }^{4}$ Missouri Baptist Medical Center, St. Louis, Missouri; ${ }^{5}$ Marshfield Clinic - Weston Center, Weston, Wisconsin;

${ }^{6}$ Zucker School of Medicine at Hofstra-Northwell, Lake Success, New York; and

${ }^{7}$ The University of Texas Health Science Center at Houston, Houston, Texas.

*These authors contributed equally to this manuscript.
}

\section{Background}

More than half of new cancer cases occur in adults aged $\geq 65$ years. ${ }^{1}$ In the United States, the older adult population is increasing rapidly, resulting in an urgent need for aging-sensitive cancer care. Older adults with cancer are at high risk for polypharmacy (ie, concurrent use of multiple medications). ${ }^{2}$ Increasing comorbidities with age, ${ }^{3,4}$ prescribing cascades (ie, initiation of a medication to treat adverse effects of another medication), ${ }^{5}$ and care fragmentation across multiple specialists ${ }^{6}$ all contribute to polypharmacy. In addition, use of complementary medicines such as vitamins and supplements is common in this population, with a prevalence of up to $80 \% .^{7}$ Polypharmacy in conjunction with chemotherapeutic and supportive care medications can increase the potential for clinically significant drug-drug interactions and adverse outcomes. ${ }^{8}$ Polypharmacy is also associated with an increased risk of taking potentially inappropriate medications (PIMs; medications with a higher risk than benefit in older adults). ${ }^{3,9}$ Previous studies have shown a high prevalence of PIMs in older adults, and particularly in older adults with cancer. ${ }^{3}$

Older adults with cancer frequently have $\geq 1$ geriatric impairments, which may affect treatment tolerance and worsen outcomes. ${ }^{10}$ Presence of these impairments can be determined using geriatric assessment (GA), which is a multidisciplinary systematic process using validated tools to assess domains such as comorbidity, functional status and physical performance, cognition, and psychologic status. ${ }^{11}$ Physical functional impairment is associated with multiple adverse outcomes, including decreased quality of life, increased chemotherapy toxicity, and increased mortality, ${ }^{12}$ and maintenance of optimal physical functioning is important for these patients. Studying polypharmacy and PIMs is particularly important in older adults with advanced cancer

See page 361 for related commentary. 
because it can highlight some distinctive issues that may not apply to other populations of older adults. Older patients with advanced cancer generally have a poor prognosis; their disease is not curable and they often prioritize physical functioning and functional independence over extending duration of life when deciding on cancer treatment. ${ }^{13}$ Moreover, these patients do not have "time to benefit" from many medications, such as statins and antidiabetic medications, which may cause more harm than benefit.

In the general older adult population, polypharmacy and PIMs are associated with physical function impairment, falls, ${ }^{14}$ and longitudinal functional decline. ${ }^{15}$ However, there are few data evaluating the association of polypharmacy and/or PIMs with physical function in older adults with cancer. Moreover, there are inconsistent cutoff values for the number of medications used to define polypharmacy in the literature. Although $\geq 5$ medications is the most commonly used definition in the literature, ${ }^{3,16}$ other cutoff values have been used, including 3, 6, 9, or 10 medications. ${ }^{17} \mathrm{~A}$ better understanding of the relationships between polypharmacy/PIMs and physical function in this population could inform the development of interventions to optimize medication use in this population. For example, previous research has demonstrated that pharmacists play a significant role in assessing medication appropriateness, with respect to quality and safety of prescribing. ${ }^{18}$ However, data about the feasibility of pharmacist-led medication review or other interventions are still lacking in older adults with advanced cancer.

The aim of this cross-sectional study was to examine the baseline association of polypharmacy and PIM with physical function in older adults with advanced cancer prior to receiving a new line of chemotherapy, and to determine a cutoff value for the number of medications most strongly associated with impairment based on several validated physical functional measures. This study builds on work studying optimal cutoffs for polypharmacy in older adults newly diagnosed with cancer ${ }^{19}$; unlike prior studies, we include only older adults with advanced cancer starting chemotherapy, who may be at the most risk of adverse outcomes from polypharmacy. We hypothesized that polypharmacy and PIMs were associated with baseline impairment in physical function in older adults with advanced cancer starting chemotherapy.

\section{Methods}

\section{Study Design}

This is a cross-sectional analysis of baseline data from a nationwide multicenter study assessing whether providing GA information plus GA-guided recommendations to community oncologists reduces chemotherapy toxicity in older adults starting a new systemic treatment regimen for advanced cancer (URCC13059; ClinicalTrials.gov identifier: NCT02054741). This study was conducted within the University of Rochester Cancer Center (URCC) NCI Community Oncology Research Program (NCORP) and approved by the Institutional Review Boards at all participating sites. Enrollment of participants started in May 2014. A polypharmacy log was completed at baseline in the primary study by a clinical research associate at each study site in collaboration with the patient. In this secondary analysis, we included patients who had complete data in their polypharmacy logs at the time of analysis, including indication, frequency, doses, and route of administration of medications $(n=439)$. Individuals with incomplete data were excluded from this analysis $(n=78)$.

\section{Participants}

Eligible patients were aged $\geq 70$ years, diagnosed with an incurable stage III/IV solid tumor or lymphoma, impaired in at least 1 geriatric domain on GA, and planning to start a new systemic cancer treatment regimen within 4 weeks. Eligible treatment regimens included cytotoxic chemotherapy drugs and therapies (such as some monoclonal antibody therapies or tyrosine kinase inhibitors) that have a similar prevalence of toxicity.

\section{Medication Review}

Prior to initiation of the new cancer treatment regimen, a polypharmacy log was completed including all regular medications (both prescription and over-the-counter [OTC] medications, including complementary medications) received by the participants within 2 weeks of study enrollment. Antineoplastic therapies and supportive care medications specifically initiated in conjunction with cancer treatment were collected in a separate log and were not included in the medication count for this analysis.

PIMs were captured using the 2015 American Geriatrics Society (AGS) Beers Criteria, ${ }^{20}$ which consists of 4 parts: (1) medications that may be potentially harmful for people aged $\geq 65$ years, (2) medications with potential drug-disease interactions, (3) 13 combinations known to cause harmful drug-drug interactions, and (4) potentially problematic medications to avoid or adjusted depending on kidney function. All parts of the 2015 AGS Beers Criteria were applied by a medical oncologist using baseline study forms (polypharmacy log, comorbidity assessment, and laboratory forms) in addition to the clinic notes of the participants.

\section{Dependent Variables}

Key dependent variables were 3 validated patientreported instruments to measure physical function in 
older adults. All 3 dependent variables were categorized as binary variables (impaired vs nonimpaired). For each instrument, difficulty performing any task assessed by the instrument was graded as an impairment for that instrument. The Katz activities of daily living (ADL) scale assesses difficulty with bathing, dressing, eating, getting in or out of bed, continence, and walking; ADL impairment is defined as inability to perform $\geq 1$ activities. $^{21}$ The instrumental activities of daily living (IADL) scale assesses activities related to independent living, such as ability to use a telephone and shopping; IADL impairment was present if patients reported needing help or being unable to perform $\geq 1$ activities. ${ }^{22}$ The Older Americans Resources and Services Physical Health (OARS PH) survey assesses difficulty with 10 items, including but not limited to physical activity and walking for long distances. OARS PH impairment was present if patients selected $\geq 1$ responses for "my health limits me a lot." ${ }^{3}$

\section{Covariates}

Covariates consisted of sociodemographic, clinical, and GA variables. Sociodemographic variables included age (continuous), sex, race (White, Black, and other), education level (less than high school, high school graduate, and some college or more), and income level $(\leq \$ 50,000$ and $>\$ 50,000$ ). Clinical variables included cancer type (gastrointestinal, lung, and other), cancer stage (stage III, stage IV, and other), comorbidities (yes or no for the presence of $\geq 1$ comorbidity that affected participant a "great deal," or $\geq 3$ that affected the participant "somewhat" based on the modified OARS comorbidity scale), ${ }^{24}$ and physician-reported Karnofsky performance score (KPS; 40-60, 70-80, and 90-100). GA variables included binary variables for impairment (yes/no) for OARS Medical Social Support (impairment defined as any question answered with "some of the time," "a little of the time," or "none of the time"), ${ }^{25}$ psychological status (impairment defined as $\geq 5$ points on the Geriatric Depression Scale or $\geq 10$ points on the Generalized Anxiety Disorder-7 scale), ${ }^{26,27}$ and nutritional status (impairment defined as $\leq 11$ points on the Mini Nutritional Assessment). ${ }^{28}$

Cognitive impairment was not included in the GA covariates because in the 2015 AGS Beers Criteria, cognitive impairment is one of the conditions used to assess drug-disease interactions to determine PIMs. For example, benzodiazepines are considered PIMs when taken by individuals with cognitive impairment. To avoid the potential for collinearity in multivariate analyses, cognitive impairment was not included as a separate variable in our models.

\section{Statistical Approach}

For each of the 3 outcome measures, we used the receiver operating characteristic (ROC) curve analyses to calculate the area under the curve (AUC) and to determine the best cutoff value for number of concomitant medications (ordinal predictor) identifying association with physical functional impairments (binary outcome). On the ROC curve, each point corresponds to a specific cutoff value of the predictor, and increasing sensitivity decreases specificity (and vice versa). ROC curves facilitate an efficient identification of patients who may be at risk for physical functional impairment through use of the Youden index. ${ }^{29}$ The Youden index, calculated as the sum of the sensitivity and specificity minus 1 , captures the performance of a specific cutoff value in classifying binary outcomes. The maximum value of the Youden index represents the maximum combination of sensitivity plus specificity on ROC curve, and it is frequently used to determine the optimal cutoff value for continuous covariates.

Next, we constructed separate multivariate logistic regression models to examine the associations of polypharmacy and PIMs with ADL, IADL, and OARS PH. For each dependent variable, we tested the association with 3 independent variables: polypharmacy-A (using $\geq 5$ concomitant medications, the most commonly used cutoff value in the literature), polypharmacy-B (using the cutoff value determined by the Youden index), and PIMs ( $\geq 1$ medications according to the 2015 AGS Beers Criteria).

All models included age, sex, race, and cancer type as covariates, because these variables have been shown in prior studies to correlate with physical function outcomes in older adults. ${ }^{30-33}$

To select other covariates for the models, we used backward stepwise procedures. An interaction term between age and sex was added to the models based on prior studies; other interaction terms were considered but were not included based on literature review and clinical expertise.

The significance criteria ( $P$ value) for entry and elimination of variables from the model $(P=.157)$ was chosen because it is close to the critical level for which the stepwise procedure is asymptotically equivalent to selection of model covariates using the Akaike information criterion (AIC). ${ }^{34}$ Two-sided $P$ values of $<.05$ were considered statistically significant. All analyses were conducted using SAS 9.4 (SAS Institute Inc) and STATA, version 13.0 (StataCorp LLP).

\section{Results}

Baseline Characteristics of the Study Sample Among 439 patients included in this analysis, the mean age was 76.9 years (SD, 5.4 years), $55 \%$ were male, $86 \%$ were white, and $82 \%$ had at least a high school education. The most common cancer type was gastrointestinal 


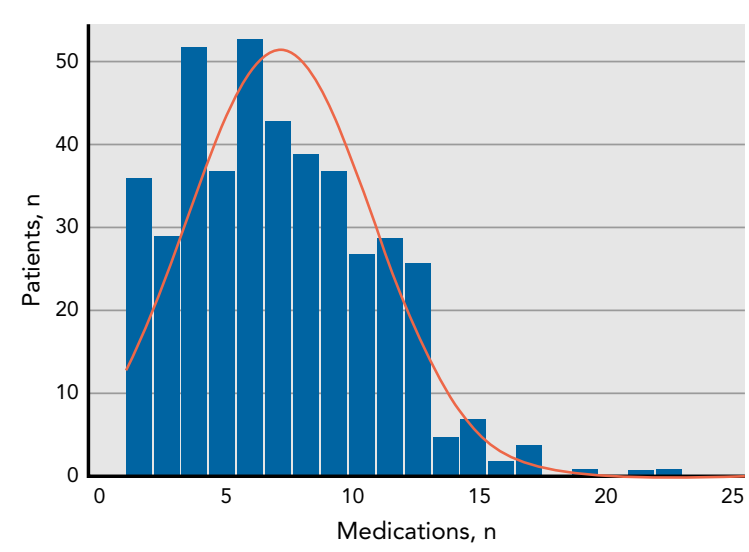

Figure 1. Histogram showing the number of patients receiving different numbers of medications in the study sample.

cancer $(34 \%)$ followed by lung cancer $(29 \%)$. In this cohort, 30\% had impairment in ADL, 55\% in IADL, and $78 \%$ in OARS PH.

\section{Prevalence of Polypharmacy and PIMs}

The mean number of medications reported was 7.1 (range, $1-23$ ), and $16 \%$ of these were complementary medications. Of the 439 patients, $71 \%$ received $\geq 5$ concurrent medications, $43 \%$ received $\geq 8$ concurrent medications, and $24 \%$ received $\geq 10$ concurrent medications (Figure 1 ).

A total of 480 medications (16\%) were considered inappropriate based on the 2015 AGS Beers Criteria. The mean number of PIMs per patient was 1.2 (range, $0-8$ ), and $62 \%$ of patients $(n=273)$ received at least one PIM. Of these patients, $96 \%$, $(n=265)$ would have been captured using only the first part of the Beers Criteria (ie, the list of medications not recommended for any adult aged $>65$ years, regardless of comorbidity or interactions). Common classes of PIMs were proton pump inhibitors (36\%), benzodiazepines (24\%), nonsteroidal anti-inflammatory drugs (NSAIDs; 18\%), and first-generation antihistamines (15\%).

ROC curves for ADL, IADL, and OARS PH demonstrated AUC results of $0.59,0.58$, and 0.59 , respectively (Figure 2). The maximum value for the Youden index was reached at $n=8$ medications for all of the examined measures, corresponding to the split of $<8$ versus $\geq 8$ medications. When OTC medications were excluded from the medication counts, the cutoffs obtained from the Youden index were 6, 5, and 8 for ADL, IADL, and OARS PH, respectively.

Table 1 shows the characteristics of the examined population according to the number of medications received ( $<8$ vs $\geq 8$ ). Patients who received $\geq 8$ concurrent medications were more likely to be female $(51 \%$ vs $40 \% ; P=.02)$ and impaired on OARS comorbidity scale ( $83 \%$ vs $56 \%$; $P<.001$ ).

\section{Association of Polypharmacy and PIMs With \\ Physical Function}

In multivariate logistic regression models, the use of $\geq 8$ medications was associated with impairment in ADL (adjusted odds ratio [aOR], 1.64; 95\% CI, 1.01-2.58) and OARS PH (aOR, 1.73; 95\% CI, 1.01-2.98), but not with IADL (aOR, 1.26; 95\% CI, 0.81-1.97). Taking $\geq 5$ concurrent medications (ie, using the most common definition for polypharmacy in the literature) was not associated with impairment of any of the physical function measures. PIMs were associated with impairment in IADL (aOR, 1.72; 95\% CI, 1.09-2.73) and OARS PH (aOR, 1.97; 95\% CI, 1.15-3.37) but not with ADL (aOR,
A

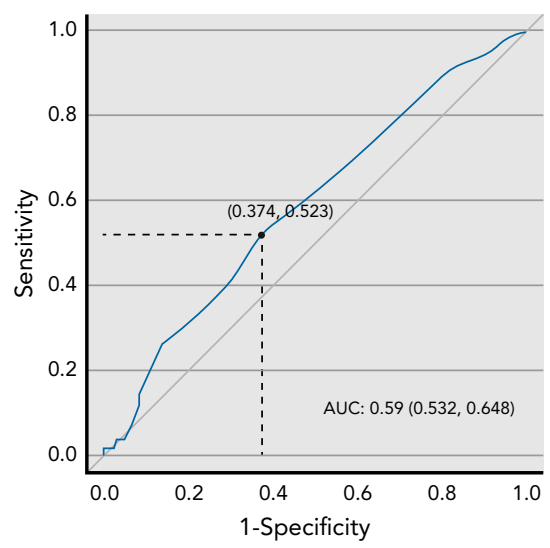

B

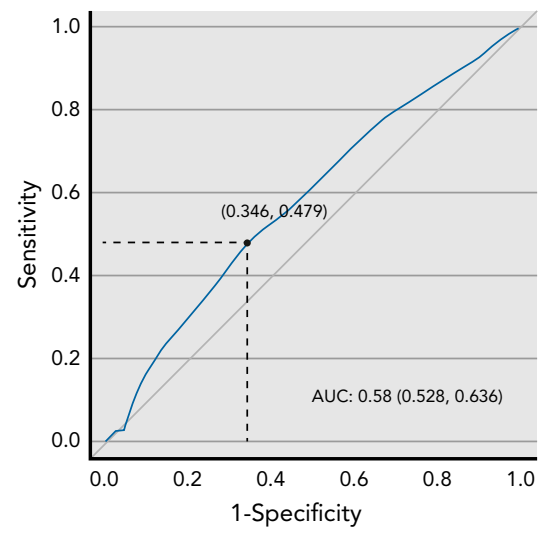

C

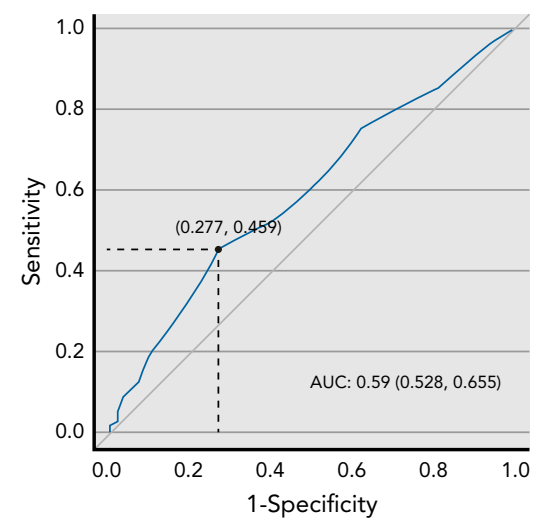

Figure 2. Receiver operating characteristic curves for the examined physical function outcomes in relation to the number of medications. AUC results for (A) activities of daily living, (B) instrumental activities of daily living, and (C) Older Americans Resources and Services Physical Health were 0.59, 0.58 , and 0.59 , respectively. The maximum value for the Youden index was reached at $n=8$ medications for all of the examined measures. Abbreviation: AUC, area under the curve. 


\begin{tabular}{|c|c|c|c|c|}
\hline Variable & n (\%) & $\begin{array}{c}<8 \\
\text { Medications } \\
\mathrm{n}(\%)\end{array}$ & $\begin{array}{c}\geq 8 \\
\text { Medications } \\
n(\%)\end{array}$ & $\begin{array}{c}P \\
\text { Value }\end{array}$ \\
\hline Total, N & 439 & 250 & 189 & \\
\hline \multicolumn{5}{|l|}{ Sociodemographic } \\
\hline Mean age (SD), y & $76.9(5.4)$ & $77.2(5.5)$ & $76.6(5.3)$ & .30 \\
\hline Sex & & & & .02 \\
\hline Male & $244(55.4)$ & $150(60.2)$ & 93 (48.9) & \\
\hline Female & $195(44.6)$ & $99(39.8)$ & 97 (51.1) & \\
\hline Race $^{a}$ & & & & .97 \\
\hline White & $375(86.0)$ & $213(86.2)$ & $162(85.7)$ & \\
\hline Black & $37(8.5)$ & $21(8.5)$ & $16(8.5)$ & \\
\hline Other & $24(5.5)$ & $13(5.3)$ & $11(5.8)$ & \\
\hline Education level ${ }^{b}$ & & & & .96 \\
\hline $\begin{array}{l}\text { Some college } \\
\text { or more }\end{array}$ & $213(48.7)$ & $122(49.2)$ & $91(48.1)$ & \\
\hline $\begin{array}{l}\text { High school } \\
\text { graduate }\end{array}$ & 145 (33.2) & $81(32.7)$ & 64 (33.9) & \\
\hline$<$ High school & 79 (18.1) & $45(18.1)$ & $34(18.0)$ & \\
\hline Income level, a USD & & & & .24 \\
\hline$>\$ 50,000$ & $200(45.9)$ & $120(48.4)$ & $80(42.6)$ & \\
\hline$\leq \$ 50,000$ & $236(54)$ & $128(51.6)$ & $108(57.4)$ & \\
\hline \multicolumn{5}{|l|}{ Clinical } \\
\hline Cancer type ${ }^{c}$ & & & & .28 \\
\hline Gastrointestinal & $148(34.4)$ & $85(34.4)$ & $63(34.2)$ & \\
\hline Lung & $125(29.1)$ & $65(26.3)$ & $60(32.6)$ & \\
\hline Other & $158(36.6)$ & 97 (39.3) & $61(33.2)$ & \\
\hline Cancer stage $^{c}$ & & & & .95 \\
\hline III & $54(12.4)$ & $30(12.2)$ & $24(13.0)$ & \\
\hline IV & $366(85)$ & $210(85.4)$ & $156(84.3)$ & \\
\hline Other ${ }^{d}$ & $11(2.6)$ & $6(2.4)$ & $5(2.7)$ & \\
\hline Comorbidities & & & & $<.001$ \\
\hline Impaired ${ }^{\mathrm{e}}$ & $297(67.7)$ & $141(56.4)$ & $156(82.5)$ & \\
\hline Nonimpaired & $142(32.3)$ & $109(43.6)$ & $33(17.5)$ & \\
\hline \multicolumn{2}{|c|}{ Physician-reported KPS ${ }^{f}$} & & & .14 \\
\hline $40-60$ & $64(14.7)$ & $33(13.4)$ & $31(16.6)$ & \\
\hline $70-80$ & $242(55.8)$ & $132(53.4)$ & $110(58.8)$ & \\
\hline $90-100$ & $128(29.5)$ & 82 (33.2) & $46(24.6)$ & \\
\hline
\end{tabular}

(continued)

1.42; 95\% CI, 0.87-2.32) (Table 2). Sensitivity analysis, using only the 265 patients receiving PIMs captured by the first part of the 2015 AGS Beers Criteria, did not alter these results.

\section{Discussion}

In this cross-sectional analysis of baseline data from a large cohort of older adults with advanced cancer

\begin{tabular}{|c|c|c|c|c|}
\hline Variable & n (\%) & $\begin{array}{c}<8 \\
\text { Medications } \\
n(\%)\end{array}$ & $\begin{array}{c}\geq 8 \\
\text { Medications } \\
n(\%)\end{array}$ & $\begin{array}{c}P \\
\text { Value }\end{array}$ \\
\hline \multicolumn{5}{|l|}{ Geriatric assessment } \\
\hline \multicolumn{2}{|c|}{ OARS Medical Social Support } & & & .48 \\
\hline Impairedg & $127(28.9)$ & $69(27.6)$ & $58(30.7)$ & \\
\hline Nonimpaired & 312 (71.1) & $181(72.4)$ & $131(69.3)$ & \\
\hline \multicolumn{2}{|c|}{ Psychological status } & & & .37 \\
\hline Impaired ${ }^{\text {h }}$ & $289(65.8)$ & $169(67.6)$ & $120(63.5)$ & \\
\hline Nonimpaired & $150(34.2)$ & $81(32.4)$ & $69(36.5)$ & \\
\hline \multicolumn{2}{|c|}{ Mini Nutritional Assessment } & & & .11 \\
\hline Impairedi & $279(63.6)$ & $151(60.4)$ & $128(67.7)$ & \\
\hline Nonimpaired & $160(36.4)$ & 99 (39.6) & $61(32.3)$ & \\
\hline
\end{tabular}

Abbreviations: KPS, Karnofsky performance score; OARS, Older Americans Resources and Services.

aMissing data: $n=3$.

bMissing data: $n=2$.

cMissing data: $n=8$.

dRefers to cancer types that have different staging system, such as small cell lung cancer.

ePresence of $\geq 1$ comorbidity that affected participant a "great deal," or $\geq 3$ that affected the participant "somewhat" based on the modified OARS comorbidity scale.

fMissing data: $n=5$

gAnswering any of the questions on OARS Medical Social Support as some of the time, a little of the time, or none of the time.

$h \geq 5$ points on the Geriatric Depression Scale or $\geq 10$ points on the Generalized Anxiety Scale-7.

i $\leq 11$ points on the Mini Nutritional Assessment.

enrolled on a clinical trial, we identified a high prevalence of polypharmacy ( $71 \%$ taking $\geq 5$ medications, $43 \%$ taking $\geq 8$ medications, and $24 \%$ taking $\geq 10$ medications) and PIMs (62\% taking medications in the 2015 AGS Beers Criteria). We found both polypharmacy $(\geq 8$ medications) and PIMs were independently associated with physical function impairments measured using validated tools. To the authors' knowledge, this is the first study demonstrating that PIMs are associated with physical function impairment in older adults with cancer. Given the cross-sectional nature of the study, however, conclusions about causality cannot be drawn.

The definition of polypharmacy varies in the literature, with $\geq 5$ medications as the most commonly used definition. ${ }^{35}$ Previous data suggest a prevalence of polypharmacy as high as $92 \%$ in older adults with cancer using this definition. ${ }^{4}$ Unlike many studies to date, ${ }^{36,37}$ we included nonprescription medications (ie, OTC) in addition to prescription medications when defining polypharmacy. Many OTC medications, including pain medications (eg, NSAIDs) and sleep aids (eg, diphenhydramine), are prevalent and considered to be PIMs; they may lead to adverse events, such as acute kidney injury, gastrointestinal bleeding, stroke, cognitive decline, and falls. ${ }^{38,39}$ Excluding OTC medications may underestimate the effects of polypharmacy and/or PIMs. 


\begin{tabular}{|c|c|c|c|}
\hline Variable & $\begin{array}{c}\text { ADL Impairment (30\%) } \\
\text { aOR }(95 \% \mathrm{Cl})\end{array}$ & $\begin{array}{l}\text { IADL Impairment (55\%) } \\
\text { aOR }(95 \% \mathrm{CI})\end{array}$ & $\begin{array}{c}\text { OARS PH Impairment (78\%) } \\
\text { aOR }(95 \% \mathrm{Cl})\end{array}$ \\
\hline \multicolumn{4}{|l|}{ Polypharmacy } \\
\hline Continuous & $1.05(0.99-1.12)$ & $1.01(0.95-1.08)$ & $1.03(0.95-1.11)$ \\
\hline$\geq 5$ medications & $1.44(0.83-2.49)$ & $1.095(0.68-1.77)$ & $1.23(0.71-2.12)$ \\
\hline \multicolumn{4}{|c|}{ Polypharmacy-B (Youden index) } \\
\hline$<8$ medications & Ref & Ref & Ref \\
\hline$\geq 8$ medications & $1.64(1.01-2.58)^{\star}$ & $1.26(0.81-1.97)$ & $1.73(1.01-2.98)^{\star}$ \\
\hline
\end{tabular}

Abbreviations: ADL, activities of daily living; aOR, adjusted odds ratio; IADL, instrumental activities of daily living; KPS, Karnofsky performance score; OARS PH, Older Americans Resources and Services Physical Health; PIM, potentially inappropriate medication.

aADL final model: included covariates were age, sex, race, cancer type, income level, and KPS.

bIADL final model: included covariates were age, sex, race, cancer type, income level, KPS, education level, and comorbidities.

'OARS PH final model: included covariates were age, sex, race, cancer type, income level, KPS, and comorbidities. $\star P<.05$.

In addition, OTC medications may interact with each other and with other prescription medications, ${ }^{38}$ including cancer therapies. ${ }^{8}$

Our study suggests that $\geq 8$ medications is the optimal cutoff value associated with physical function impairments in older adults with cancer. A previous cross-sectional analysis of 385 older adults with various types of cancers suggested multiple cutoff values of polypharmacy (4.5-6.5) in relation to falls, frailty, physical function, and KPS. ${ }^{19}$ Another cross-sectional analysis of 1,705 community-dwelling men also identified several cutpoints of polypharmacy (3.5-6.5) in relation to multiple adverse outcomes. In that study, polypharmacy was associated with falls, frailty, and mortality, ${ }^{36}$ but only prescription medications were counted. Given that the medication counts for our analysis included OTC and "self-prescribed" medications, a higher threshold is not an unexpected finding.

Our study indicated that polypharmacy (defined as the use of $\geq 8$ medications) is associated with physical function impairments in older adults with cancer. A retrospective cohort of 837 patients with breast and colorectal cancers found that polypharmacy ( $\geq 5$ medications) was associated with impairment in ADL and IADL ${ }^{40}$ Associations between polypharmacy and physical function are likely multifactorial. Polypharmacy may reflect a high burden of comorbidities in this population. We included a measure of patient-reported comorbidity interference as a covariate, but this may not have captured the full impact of comorbidity on physical functioning. Polypharmacy may also contribute to adverse events and potential drug-drug interactions, which can worsen physical function. Finally, patients with poorer physical function may take more medications for symptoms, including OTC medications.

We demonstrated a high prevalence of PIMs (62\%) in this cohort. PIM prevalence ranged from $47 \%$ to $66 \%$ in a previously reported cohort of older adults with breast, colorectal, and prostate cancers. ${ }^{41}$ PIMs are associated with physical function impairments in our study; they may lead to adverse effects that impact physical function (eg, first-generation antihistamines can cause drowsiness, delirium, and falls, which may precipitate physical and functional decline)..$^{42}$ Moreover, decreased physiologic resilience and altered drug metabolism in aging can make older adults more susceptible to medication adverse events. ${ }^{43}$ Findings on the association of PIMs with other health outcomes are mixed in older adults with cancer. ${ }^{44,45}$ In patients with breast or colorectal cancer receiving adjuvant chemotherapy, there was no association between PIMs and adverse health outcomes, including emergency department visits, hospitalizations, or death. ${ }^{46}$

Understanding the relationships between polypharmacy, PIMs, and outcomes of older patients with cancer could help guide interventions. For example, "deprescribing" (the planned discontinuation of medications) is an intervention strategy that has been investigated in community-dwelling older adults to optimize medication use..$^{47}$ Data for deprescribing in older patients with cancer are very limited, and it is unclear how this strategy could best be implemented into oncology clinic 
workflows. ${ }^{48}$ Pharmacists have been effectively used in the clinic to identify polypharmacy and PIMs, ${ }^{3}$ and a pharmacist-led deprescribing intervention in older adults with cancer has been shown to be feasible. ${ }^{49}$ Providing information to oncologists about patients' medications increases in-clinic conversations about medication management, ${ }^{50}$ but it is unclear whether this is sufficient to drive deprescribing by oncologists. Randomized studies investigating deprescribing are needed to identify interventions that may improve adverse outcomes in older adults with cancer and polypharmacy and/or PIMs.

Our study has several strengths, including the use of a large sample of older adults with advanced cancer receiving treatment in community oncology (ie, realworld) practices. We also used validated instruments to assess physical function.

This study also has several limitations. First, our findings should be interpreted carefully because of the cross-sectional design. We are unable to suggest causal hypotheses in our analyses because temporality of association is a strong criterion for causality. Second, medications were captured from a medication log, which did not take account into medication adherence. Third, this study used total count of all medications, which is a crude measure; however, it is easy to perform in a clinical setting, and it is not always feasible to determine which medications are prescribed versus OTC. Fourth, we were not able to consider other inappropriate prescribing parameters, such as indication, dosage, directions for use, and duration of use. Fifth, medications were captured at one time point, thus we were not able to examine the potential changes over the course of cancer treatment; medications specifically initiated as supportive care medications for cancer treatment were not included in our analyses. Finally, AUC results were only slightly better than chance to differentiate different functional outcomes by polypharmacy (AUC, 0.59, 0.58, and 0.59 for ADL, IADL, and OARS $\mathrm{PH}$, respectively).
This suggests that functional outcomes are influenced by additional variables other than polypharmacy that could include cognition impairment, ${ }^{51}$ malnutrition, ${ }^{52}$ and comorbidities. ${ }^{51}$

\section{Conclusions}

Our findings showed that polypharmacy (the use of $\geq 8$ medications) and use of PIMs ( $\geq 1$ medications included in the 2015 AGS Beers Criteria) were associated with physical function impairments in older adults with advanced cancer receiving cancer treatment. This highlights the importance of comprehensive evaluation of medication use, including OTC and complementary medications, in older adults with cancer. Although causality was not addressed by this crosssectional study, future work can determine whether optimizing medication use may reduce the risk of functional decline, thereby improving quality of life and survival. ${ }^{12}$ Future studies should prospectively evaluate the effect of polypharmacy and PIMs on the longitudinal risk of physical functional decline in older adults with cancer and determine whether prospective interventions such as deprescribing can improve outcomes in these patients.

Submitted January 11, 2020; accepted for publication July 23, 2020. Published online January 22, 2021.

Author contributions: Study concept and design: Mohamed, Ramsdale, Loh, Mohile. Collection and/or assembly of data: Mohamed, Ramsdale, Obrecht, Xu. Data analysis and interpretation: Mohamed, Ramsdale, Loh, Xu, Patil. Manuscript writing, review, and editing: All authors.

Disclosures: Dr. Loh has disclosed that she is a consultant for Pfizer and Seattle Genetics. Dr. Holmes has disclosed that she receives funding from the Cancer Prevention and Research Institute of Texas and Blue Cross/Blue Shield Health Care Service Corporation. The remaining authors have disclosed that they have not received any financial consideration from any person or organization to support the preparation, analysis, results, or discussion of this article.

Correspondence: Mostafa R. Mohamed, MBBCh, MSc, James P. Wilmot Cancer Center, University of Rochester Medical Center, 601 Elmwood Avenue, Box 704, Rochester, NY 14642. Email: mostafa_mohamed@urmc.rochester.edu

\section{References}

1. Siegel RL, Miller KD, Jemal A. Cancer statistics, 2017. CA Cancer J Clin 2017:67:7-30.

2. Sharma M, Loh KP, Nightingale G, et al. Polypharmacy and potentially inappropriate medication use in geriatric oncology. J Geriatr Oncol 2016; 7:346-353.

3. Nightingale G, Hajjar E, Swartz K, et al. Evaluation of a pharmacist-led medication assessment used to identify prevalence of and associations with polypharmacy and potentially inappropriate medication use among ambulatory senior adults with cancer. J Clin Oncol 2015;33: 1453-1459.

4. Maggiore RJ, Gross CP, Hurria A. Polypharmacy in older adults with cancer. Oncologist 2010;15:507-522.

5. Rochon PA, Gurwitz JH. The prescribing cascade revisited. Lancet 2017; 389:1778-1780.

6. Clyne B, Cooper JA, Hughes CM, et al. 'Potentially inappropriate or specifically appropriate?' Qualitative evaluation of general practitioners

views on prescribing, polypharmacy and potentially inappropriate prescribing in older people. BMC Fam Pract 2016;17:109.

7. Richardson MA, Sanders T, Palmer JL, et al. Complementary/alternative medicine use in a comprehensive cancer center and the implications for oncology. J Clin Oncol 2000;18:2505-2514

8. Popa MA, Wallace KJ, Brunello A, et al. Potential drug interactions and chemotoxicity in older patients with cancer receiving chemotherapy. J Geriatr Oncol 2014;5:307-314.

9. Cannon KT, Choi MM, Zuniga MA. Potentially inappropriate medication use in elderly patients receiving home health care: a retrospective data analysis. Am J Geriatr Pharmacother 2006;4:134-143.

10. Sarfati D, Koczwara B, Jackson C. The impact of comorbidity on cancer and its treatment. CA Cancer J Clin 2016;66:337-350.

11. Extermann M, Aapro M, Bernabei R, et al. Use of comprehensive geriatric assessment in older cancer patients: recommendations from the task force on CGA of the International Society of 
Geriatric Oncology (SIOG). Crit Rev Oncol Hematol 2005;55 241-252.

12. Chen $\mathrm{C}$, Sia I, Ma HM, et al. The synergistic effect of functional status and comorbidity burden on mortality: a 16-year survival analysis. PLoS One 2014;9:e106248.

13. Mohile SG, Dale W, Somerfield MR, et al. Practical assessment and management of vulnerabilities in older patients receiving chemotherapy: ASCO Guideline for Geriatric Oncology. J Clin Oncol 2018;36: 2326-2347.

14. Fried TR, O'Leary J, Towle $\mathrm{V}$, et al. Health outcomes associated with polypharmacy in community-dwelling older adults: a systematic review. J Am Geriatr Soc 2014;62:2261-2272.

15. Jyrkkä J, Enlund $H$, Lavikainen $P$, et al. Association of polypharmacy with nutritional status, functional ability and cognitive capacity over a threeyear period in an elderly population. Pharmacoepidemiol Drug Saf 2011; 20:514-522.

16. Turner JP, Shakib S, Singhal N, et al. Prevalence and factors associated with polypharmacy in older people with cancer. Support Care Cancer 2014:22:1727-1734.

17. Mohamed MR, Ramsdale E, Loh KP, et al. Associations of polypharmacy and inappropriate medications with adverse outcomes in older adults with cancer: a systematic review and meta-analysis. Oncologist 2020;25 e94-108.

18. Muijrers PE, Knottnerus JA, Sijbrandij J, et al. Changing relationships: attitudes and opinions of general practitioners and pharmacists regarding the role of the community pharmacist. Pharm World Sci 2003; 25:235-241.

19. Turner JP, Jamsen KM, Shakib S, et al. Polypharmacy cut-points in older people with cancer: how many medications are too many? Support Care Cancer 2016;24:1831-1840.

20. American Geriatrics Society 2015 Beers Criteria Update Expert Panel. American Geriatrics Society 2015 Updated Beers Criteria for potentially inappropriate medication use in older adults. J Am Geriatr Soc 2015;63: 2227-2246.

21. Brorsson B, Asberg KH. Katz index of independence in ADL. Reliability and validity in short-term care. Scand J Rehabil Med 1984;16:125-132.

22. Lawton MP, Brody EM. Assessment of older people: self-maintaining and instrumental activities of daily living. Gerontologist 1969;9:179-186.

23. Whitelaw NA, Liang J. The structure of the OARS physical health measures. Med Care 1991;29:332-347.

24. Fillenbaum GG, Smyer MA. The development, validity, and reliability of the OARS multidimensional functional assessment questionnaire. J Gerontol 1981;36:428-434.

25. Burholt $\mathrm{V}$, Windle $G$, Ferring $D$, et al. Reliability and validity of the Older Americans Resources and Services (OARS) social resources scale in six European countries. J Gerontol B Psychol Sci Soc Sci 2007;62:S371-379.

26. Weintraub D, Oehlberg KA, Katz IR, et al. Test characteristics of the 15item geriatric depression scale and Hamilton depression rating scale in Parkinson disease. Am J Geriatr Psychiatry 2006;14:169-175.

27. Wild B, Eckl A, Herzog W, et al. Assessing generalized anxiety disorder in elderly people using the GAD-7 and GAD-2 scales: results of a validation study. Am J Geriatr Psychiatry 2014;22:1029-1038.

28. Montejano Lozoya R, Martínez-Alzamora N, Clemente Marín G, et al. Predictive ability of the Mini Nutritional Assessment Short Form (MNA-SF) in a free-living elderly population: a cross-sectional study. PeerJ 2017;5: e3345.

29. Ruopp MD, Perkins NJ, Whitcomb BW, et al. Youden index and optimal cut-point estimated from observations affected by a lower limit of detection. Biom J 2008;50:419-430.

30. Owusu C, Schluchter M, Koroukian SM, et al. Racial disparities in functional disability among older women with newly diagnosed nonmetastatic breast cancer. Cancer 2013;119:3839-3846.

31. Weaver $\mathrm{KE}$, Leach $\mathrm{CR}$, Leng $\mathrm{X}$, et al. Physical functioning among women 80 years of age and older with and without a cancer history. J Gerontol A Biol Sci Med Sci 2016;71(Suppl 1):S23-30.
32. Petrick JL, Reeve BB, Kucharska-Newton AM, et al. Functional status declines among cancer survivors: trajectory and contributing factors. J Geriatr Oncol 2014;5:359-367.

33. den Ouden ME, Schuurmans MJ, Mueller-Schotte S, et al. Identification of high-risk individuals for the development of disability in activities of daily living. A ten-year follow-up study. Exp Gerontol 2013;48:437-443.

34. Akaike H. A new look at the statistical-model identification. IEEE Transactions on Automatic Control 1974;19:716-723.

35. Masnoon N, Shakib S, Kalisch-Ellett L, et al. What is polypharmacy? A systematic review of definitions. BMC Geriatr 2017;17:230.

36. Gnjidic D, Hilmer SN, Blyth FM, et al. Polypharmacy cutoff and outcomes: five or more medicines were used to identify community-dwelling older men at risk of different adverse outcomes. J Clin Epidemiol 2012;65: 989-995.

37. Elliot K, Tooze JA, Geller R, et al. The prognostic importance of poly pharmacy in older adults treated for acute myelogenous leukemia (AML). Leuk Res 2014;38:1184-1190.

38. Roumie CL, Griffin MR. Over-the-counter analgesics in older adults: a call for improved labelling and consumer education. Drugs Aging 2004;21: 485-498.

39. Abraham $\mathrm{O}$, Schleiden L, Albert SM. Over-the-counter medications containing diphenhydramine and doxylamine used by older adults to improve sleep. Int J Clin Pharm 2017;39:808-817.

40. van Abbema D, van Vuuren $A$, van den Berkmortel $F$, et al. Functional status decline in older patients with breast and colorectal cancer after cancer treatment: a prospective cohort study. J Geriatr Oncol 2017;8: 176-184.

41. Feng $X$, Higa GM, Safarudin F, et al. Potentially inappropriate medication use and associated healthcare utilization and costs among older adults with colorectal, breast, and prostate cancers. J Geriatr Oncol 2019;10: 698-704.

42. Kalpaklioglu F, Baccioglu A. Efficacy and safety of $\mathrm{H} 1$-antihistamines: an update. Antiinflamm Antiallergy Agents Med Chem 2012;11:230-237.

43. Rosenthal RA, Kavic SM. Assessment and management of the geriatric patient. Crit Care Med 2004;32(4 Suppl):S92-105.

44. Maggiore RJ, Dale W, Gross CP, et al. Polypharmacy and potentially inappropriate medication use in older adults with cancer undergoing chemotherapy: effect on chemotherapy-related toxicity and hospitalization during treatment. J Am Geriatr Soc 2014;62: 1505-1512.

45. Lin RJ, Ma H, Guo R, et al. Potentially inappropriate medication use in elderly non-Hodgkin lymphoma patients is associated with reduced survival and increased toxicities. Br J Haematol 2018;180:267-270.

46. Karuturi MS, Holmes HM, Lei X, et al. Potentially inappropriate medication use in older patients with breast and colorectal cancer. Cancer 2018;124 3000-3007.

47. Page AT, Clifford RM, Potter K, et al. The feasibility and effect of deprescribing in older adults on mortality and health: a systematic review and meta-analysis. Br J Clin Pharmacol 2016;82:583-623.

48. Turner JP, Shakib S, Bell JS. Is my older cancer patient on too many medications? J Geriatr Oncol 2017;8:77-81.

49. Whitman A, DeGregory K, Morris A, et al. Pharmacist-led medication assessment and deprescribing intervention for older adults with cancer and polypharmacy: a pilot study. Support Care Cancer 2018;26: 4105-4113.

50. Ramsdale E, Lemelman T, Loh K, et al. Geriatric assessment-driven polypharmacy discussions between oncologists, older patients with cancer, and their caregivers. J Geriatr Oncol 2018;9:534-539.

51. Hajek $\mathrm{A}$, König $\mathrm{HH}$. Longitudinal predictors of functional impairment in older adults in Europe - evidence from the Survey of Health, Ageing and Retirement in Europe. PLoS One 2016;11:e0146967.

52. Kiesswetter E, Pohlhausen S, Uhlig K, et al. Malnutrition is related to functional impairment in older adults receiving home care. J Nutr Health Aging 2013;17:345-350. 